ELECTRONIC RESEARCH ANNOUNCEMENTS OF THE AMERICAN MATHEMATICAL SOCIETY

Volume 9, Pages 80-87 (September 15, 2003)

S $1079-6762(03) 00114-8$

\title{
ALGEBRAS OF PSEUDODIFFERENTIAL OPERATORS ON COMPLETE MANIFOLDS
}

\author{
BERND AMMANN, ROBERT LAUTER, AND VICTOR NISTOR
}

(Communicated by Michael E. Taylor)

\begin{abstract}
In several influential works, Melrose has studied examples of noncompact manifolds $M_{0}$ whose large scale geometry is described by a Lie algebra of vector fields $\mathcal{V} \subset \Gamma(M ; T M)$ on a compactification of $M_{0}$ to a manifold with corners $M$. The geometry of these manifolds - called "manifolds with a Lie structure at infinity" - was studied from an axiomatic point of view in a previous paper of ours. In this paper, we define and study an algebra $\Psi_{1,0, \mathcal{V}}^{\infty}\left(M_{0}\right)$ of pseudodifferential operators canonically associated to a manifold $M_{0}$ with a Lie structure at infinity $\mathcal{V} \subset \Gamma(M ; T M)$. We show that many of the properties of the usual algebra of pseudodifferential operators on a compact manifold extend to the algebras that we introduce. In particular, the algebra $\Psi_{1,0, \mathcal{V}}^{\infty}\left(M_{0}\right)$ is a "microlocalization" of the algebra $\operatorname{Diff}_{\mathcal{V}}^{*}(M)$ of differential operators with smooth coefficients on $M$ generated by $\mathcal{V}$ and $\mathcal{C}^{\infty}(M)$. This proves a conjecture of Melrose (see his ICM 90 proceedings paper).
\end{abstract}

\section{INTRODUCTION}

In 17, Melrose has formulated a far reaching program to study the analytic properties of geometric differential operators on an open manifold $M_{0}$, provided that its large scale geometry is controlled by a Lie algebra of vector fields $\mathcal{V}$ on a compactification $M \supset M_{0}$. Typically, $M$ is a manifold with corners, $M_{0}=M \backslash \partial M$ is obtained by removing all faces of $M$, and the given Lie algebra of vector fields $\mathcal{V} \subset \Gamma(M ; T M)$ satisfies a number of axioms (see Section 1). This structure leads to complete metrics of bounded curvature on $M_{0}$, and $\partial M$ is the "boundary at infinity." For example, manifolds with asymptotically Euclidean, asymptotically hyperbolic, or asymptotically complex hyperbolic ends are obtained in this way.

An important ingredient in Melrose's program mentioned above is to define a suitable pseudodifferential calculus on $M_{0}$ adapted in a certain sense to $(M, \mathcal{V})$. Melrose calls this pseudodifferential calculus a "microlocalization of $\operatorname{Diff}_{\mathcal{V}}^{*}(M)$," where $\operatorname{Diff}_{\mathcal{V}}^{*}(M)$ is the algebra of differential operators on $M$ generated by $\mathcal{V}$ and $\mathcal{C}^{\infty}(M)$.

Received by the editors April 24, 2003.

2000 Mathematics Subject Classification. Primary 58J40; Secondary 58H05, 65R20.

Key words and phrases. Differential operator, pseudodifferential operator, principal symbol, conormal distribution, Riemannian manifold, Lie algebra, exponential map.

Ammann was partially supported by the European Contract Human Potential Program, Research Training Networks HPRN-CT-2000-00101 and HPRN-CT-1999-00118; Nistor was partially supported by NSF Grants DMS 99-1981 and DMS 02-00808. Manuscripts available from http://www.math.psu.edu/nistor/.

(C)2003 American Mathematical Society 
In [19] and several other papers, Melrose and his collaborators have completed this program in several important cases [6, 9, 10, 13, 14, 15, 16, 18, 19, 20, 21, [22] [32]. One of the main points is that the geometric operators on manifolds with a Lie structure at infinity identify with degenerate differential operators on the compactification $M$. This type of differential operators appear naturally, for example, in the study of boundary value problems on manifolds with singularities. Results in this direction were obtained also by Schulze and his collaborators, who typically worked in the framework of the Boutet de Monvel algebras. See [26, 27. and the references therein. See also [11, 24, 25].

It is desirable to present all these cases in a unified setting and to extend the results to a larger class of manifolds, namely the class of "manifolds with a Lie structure at infinity." These are open manifolds $M_{0}$ which are topologically the interior of a compact manifold $M$ with corners, and the geometry of $M_{0}$ near $\partial M$ is described by a Lie algebra of vector fields $\mathcal{V} \subset \Gamma(T M)$ satisfying certain axioms (see Section 1 for details). The geometrical properties of these manifolds were studied in [1]. Here we introduce an algebra $\Psi_{1,0, \mathcal{V}}^{\infty}\left(M_{0}\right)$ of pseudodifferential operators on $M_{0}$ that is canonically associated to the manifold with a Lie structure at infinity $M_{0}$. Then we show that this algebra "microlocalizes" $\operatorname{Diff}_{\mathcal{V}}^{*}(M)$ in the sense that $\operatorname{Diff}_{\mathcal{V}}^{*}(M)$ is the algebra of all differential operators in $\Psi_{1,0, \mathcal{V}}^{\infty}\left(M_{0}\right)$ and that $\Psi_{1,0, \mathcal{V}}^{\infty}\left(M_{0}\right)$ has the usual symbolic properties of algebras of pseudodifferential operators on a compact manifold. We also show that the algebra $\Psi_{1,0, \mathcal{V}}^{\infty}\left(M_{0}\right)$ is invariant under the diffeomorphisms of $M_{0}$ obtained by exponentiating the vector fields $X \in \mathcal{V}$ and under conjugation with complex powers of the functions that define the faces of the compactification $M$ of $M_{0}$.

The explicit construction of the algebra $\Psi_{1,0, \mathcal{V}}^{\infty}\left(M_{0}\right)$ microlocalizing $\operatorname{Diff} \mathcal{V}\left(M_{0}\right)$ in the sense of [17] is, roughly, as follows. First, $\mathcal{V}$ defines an extension of $T M_{0}$ to a vector bundle $A \rightarrow M\left(M_{0}=M \backslash \partial M\right)$. Denote $V_{r}:=\{d(x, y)<r\} \subset M_{0}^{2}$ and $(A)_{r}=\{v \in A,\|v\|<r\}$. Let $r>0$ be less than the injectivity radius of $M_{0}$ and $V_{r} \ni(x, y) \mapsto(x, \tau(x, y)) \in(A)_{r}$ be a local inverse of the Riemannian exponential map $T M_{0} \ni v \mapsto \exp _{x}(-v) \in M_{0} \times M_{0}$. Let $\chi$ be a smooth function on $A$ with support in $(A)_{r}, \chi=1$ on $(A)_{r / 2}$. For any $a \in S_{1,0}^{m}\left(A^{*}\right)$, we define

$$
\left[a_{\chi}(D) u\right](x)=(2 \pi)^{-n} \int_{M_{0}}\left(\int_{T_{x}^{*} M_{0}} e^{i \tau(x, y) \cdot \eta} \chi(x, \tau(x, y)) a(x, \eta) u(y) d \eta\right) d y .
$$

The algebra $\Psi_{1,0, \mathcal{V}}^{\infty}\left(M_{0}\right)$ is then generated linearly by the operators $a_{\chi}(D)$ and $b_{\chi}(D) \exp \left(X_{1}\right) \ldots \exp \left(X_{k}\right), a \in S^{\infty}\left(A^{*}\right), b \in S^{-\infty}\left(A^{*}\right)$, and $X_{j} \in \mathcal{V}$. (We need to introduce the operators $b_{\chi}(D) \exp \left(X_{1}\right) \ldots \exp \left(X_{k}\right)$, where $\exp \left(X_{j}\right)$ is the exponential of the vector field $X_{j}$, to make our space of operators closed under products.) The definition of the operators of order $-\infty$ is in fact the crucial part of this construction.

In [17, Melrose outlined the construction of a pseudodifferential calculus on manifolds with Lie structure at infinity provided certain manifolds with corners (blow-ups) can be constructed. (See also [31].) Our approach owes a lot to his approach when it comes to proving that $\Psi_{1,0, \mathcal{V}}^{\infty}\left(M_{0}\right)$ are algebras, but we replace the blow-ups with groupoids, using also a deep result of Crainic and Fernandes [4] on the integration of Lie algebroids. (However, to prove the original form of the conjecture from [17], the earlier results of [23] also suffice.)

This paper is an announcement. Complete proofs will be published in [2]. 
Acknowledgements: V. N. would like to thank the Institute Erwin Schrödinger in Vienna, where part of this work was completed. We also thank A. Vasy who has contributed in several ways to the results in this paper.

\section{Manifolds With Lie struCture at infinity}

For the convenience of the reader, let us recall the definition of Riemannian manifolds with a Lie structure at infinity and some of their basic properties 1 .

In the sequel, by a manifold we shall always understand a $C^{\infty}$-manifold with corners, whereas a smooth manifold is a $C^{\infty}$-manifold without corners. By definition, every point $p$ in a manifold with corners $M$ has a coordinate neighborhood diffeomorphic to $[0, \infty)^{k} \times \mathbb{R}^{n-k}$ such that the transition functions are smooth up to the boundary. We then call $p$ a point of boundary depth at most $k$ and write $\operatorname{codim}(p) \leq k$. Points $p$ with $\operatorname{codim}(p) \leq k$ but not $\operatorname{codim}(p) \leq k-1$ are said to be of boundary depth $k$. (This terminology is in agreement with the terminology for stratified spaces, if we stratify a manifold with corners by its open subfaces; see [12.) We denote by $\partial M$ the union of all non-trivial faces of $M$. Usually, we write $M_{0}$ for the interior of $M$, i.e., $M_{0}:=M \backslash \partial M$.

A Lie subalgebra $\mathcal{V} \subseteq \Gamma(M ; T M)$ of the Lie algebra of all smooth vector fields on $M$ is said to be a structural Lie algebra of vector fields provided it is a finitely generated, projective $\mathcal{C}^{\infty}(M)$-module and each $V \in \mathcal{V}$ is tangent to all hyperfaces of $M$. (We shall denote the sections of a vector bundle $V \rightarrow X$ by $\Gamma(X ; V)$, unless $X$ is understood, in which case we shall write simply $\Gamma(V)$.) By the Serre-Swan theorem [8], there exists a smooth vector bundle $A=A_{\mathcal{V}} \rightarrow M$ together with a natural map $\varrho=\varrho \mathcal{V}: A \rightarrow T M$ making the following diagram commutative

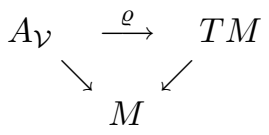

and such that $\mathcal{V}=\varrho\left(\Gamma\left(A_{\mathcal{V}}\right)\right)$.

Definition 1.1. A Lie structure at infinity on a smooth manifold $M_{0}$ is a triple $\left(M_{0}, M, \mathcal{V}\right)$, where $M$ is a compact manifold, possibly with corners, and $\mathcal{V} \subset$ $\Gamma(M ; T M)$ is a structural Lie algebra of vector fields on $M$ with the following properties:

(a) $\quad M_{0}$ is diffeomorphic to the interior $M \backslash \partial M$ of $M$;

(b) If $\varrho: A \rightarrow T M$ is the anchor map defined by diagram (2), then $\varrho$ restricts to an isomorphism $\left.\left.A\right|_{M \backslash \partial M} \rightarrow T M\right|_{M \backslash \partial M}$.

Note that for a given manifold $M_{0}$ in general there can exist many Lie structures at infinity. Examples of Lie structures at infinity were discussed in [1].

From now on, we identify $M_{0}$ with $M \backslash \partial M$ and $\left.A\right|_{M_{0}}$ with $T M_{0}$. Because $A$ and $\mathcal{V}$ determine each other up to isomorphism, we sometimes specify a Lie structure at infinity on $M$ by the pair $(M, A)$. Also, it follows that $\varrho: \Gamma(M ; A) \rightarrow \Gamma(M ; T M)$ is injective, so we shall identify $\Gamma(M ; A)$ with $\mathcal{V}=\varrho(\Gamma(M ; A))$.

Elements in the enveloping algebra $\operatorname{Diff}_{\mathcal{V}}^{*}(M)$ of $\mathcal{V}$ are called $\mathcal{V}$-differential operators on $M$. By the injectivity of the induced structural map $\varrho_{\mathcal{V}}: \Gamma\left(A_{\mathcal{V}}\right) \rightarrow \Gamma(T M)$, the algebra of $\mathcal{V}$-differential operators can be realized as a subalgebra of all differential operators on $M$; in particular, they act continuously on the space $\mathcal{C}^{\infty}(M)$. Moreover, the order of differential operators induces a filtration $\operatorname{Diff}_{\mathcal{V}}^{m}(M), m \in \mathbb{Z}_{+}$, 
on the algebra $\operatorname{Diff}_{\mathcal{V}}^{*}(M)$. Since $\operatorname{Diff}_{\mathcal{V}}^{*}(M)$ is a $\mathcal{C}^{\infty}(M)$-module, we can introduce $\mathcal{V}$ differential operators acting between sections of smooth vector bundles $E, F \rightarrow M$, $E, F \subset M \times \mathbb{C}^{N}$ by

$$
\operatorname{Diff}_{\mathcal{V}}^{*}(M ; E, F):=e_{F} M_{N}\left(\operatorname{Diff}_{\mathcal{V}}^{*}(M)\right) e_{E},
$$

where $e_{E}, e_{F} \in M_{N}\left(\mathcal{C}^{\infty}(M)\right)$ are the projections onto $E$ and, respectively, $F$. It follows that $\operatorname{Diff}_{\mathcal{V}}^{*}(M ; E, E)=: \operatorname{Diff}_{\mathcal{V}}^{*}(M ; E)$ is an algebra that is closed under adjoints and contains all geometric operators on $M_{0}$ that are associated to a metric on $M_{0}$ that comes from a metric on $A$. (See [1].)

Since any metric on $A$ induces a natural metric on $T M_{0}=\left.A\right|_{M_{0}}$, we obtain the following definition.

Definition 1.2. A manifold $M_{0}$ with a Lie structure at infinity $(M, A)$ and with metric $g_{0}$ on $M_{0}$ obtained by restricting a metric $g$ from $A$ to $T M_{0}$ is called a Riemannian manifold with a Lie structure at infinity.

The geometry of Riemannian manifolds $\left(M_{0}, g_{0}\right)$ with a Lie structure at infinity has been studied in [1]. For instance, $\left(M_{0}, g_{0}\right)$ is necessarily of infinite volume and complete. Moreover, all the covariant derivatives of the Riemannian curvature tensor are bounded. Under additional mild assumptions, we also know that the injectivity radius $\operatorname{injrad}(p)$, viewed as a function depending on $p \in M$, is bounded below by a positive constant, or, equivalently, $\left(M_{0}, g_{0}\right)$ is of bounded geometry in the sense of [28] and references therein. We shall denote by $r_{0}:=\inf _{p} \operatorname{injrad}(p)$ the injectivity radius of $M_{0}$.

On a Riemannian manifold $M_{0}$ with a uniform structure at infinity $(M, A)$, the exponential map $\exp _{p}: T M_{0} \rightarrow M_{0}$ is well defined for all $p \in M_{0}$ and extends to a differentiable map $\exp _{p}: A_{p} \rightarrow M$ depending smoothly on $p \in M$. A convenient way to introduce the exponential map is via the geodesic spray, as done in [1]. Similarly, any vector field $X \in \Gamma(A)$ is integrable and will map any (connected) face to itself. The resulting diffeomorphism of $M_{0}$ will be denoted $\psi_{X}$.

We shall also assume from now on that $r_{0}$, the injectivity radius of $\left(M_{0}, g_{0}\right)$, is positive.

\section{KOHN-NIRENBERG QUANTIZATION AND PSEUDODIFFERENTIAL OPERATORS}

We now introduce the algebras $\Psi_{c l, \mathcal{V}}\left(M_{0}\right)$ and $\Psi_{1,0, \mathcal{V}}\left(M_{0}\right)$ of pseudodifferential operators on $M_{0}$ adapted to the Lie structure at infinity $(M, \mathcal{V})$. We also state some of their main properties.

2.1. Riemann-Weyl fibration. Fix now a Riemannian metric $g$ on the bundle $A$, and let $g_{0}=\left.g\right|_{M_{0}}$ be its restriction to the interior $M_{0}$ of $M$, defined in view of the identification $\left.A\right|_{M_{0}}=T M_{0}$. We shall use this metric to trivialize all density bundles on $M$. Denote by $\pi: T M_{0} \rightarrow M_{0}$ the natural projection.

Define

$$
\Phi: T M_{0} \longrightarrow M_{0} \times M_{0}, \quad \Phi(v):=\left(x, \exp _{x}(-v)\right), x=\pi(v) .
$$

Recall that, for $v \in T_{x} M$, we have $\exp _{x}(v)=\gamma_{v}(1)$, where $\gamma_{v}$ is the unique geodesic with $\gamma_{v}(0)=\pi(v)=x$ and $\gamma_{v}^{\prime}(0)=v$. It is known that there is an open neighborhood $U$ of the zero-section $M_{0}$ in $T M_{0}$ such that $\left.\Phi\right|_{U}$ is a diffeomorphism onto an open neighborhood $V$ of the diagonal $\Delta_{M_{0}} \subseteq M_{0} \times M_{0}$. 
To fix notation, let $E$ be a vector bundle with a norm $\|\cdot\|$. We shall denote by $(E)_{r}$ the set of all vectors $v$ of $E$ with norm $\|v\|<r$. Our assumption that the injectivity radius $r_{0}$ of $M_{0}$ is positive gives that, for $0<r \leq r_{0}$, the restriction $\left.\Phi\right|_{\left(T M_{0}\right)_{r}}$ is a diffeomorphism onto an open neighborhood $V_{r}$ of the diagonal $\Delta_{M_{0}}$. We continue, by slight abuse of notation, to write $\Phi$ for that restriction. Following [5], we shall call $\Phi$ a Riemann-Weyl fibration. However, note that the Riemann-Weyl fibrations are defined in a slightly different way in [5]; the difference will be of no importance for us. The inverse of $\Phi$ is given by

$$
M_{0} \times M_{0} \supseteq V_{r} \ni(x, y) \longmapsto(x, \tau(x, y)) \in\left(T M_{0}\right)_{r},
$$

where $\tau(x, y) \in T_{x} M_{0}$ is the tangent vector at $x$ to the shortest geodesic $\gamma:[0,1] \rightarrow$ $M$ such that $\gamma(0)=x$ and $\gamma(1)=y$.

We shall denote by $S_{1,0}^{m}(E)$ the space of symbols of order $m$ and type $(1,0)$ on $E$ (in Hörmander's sense) and by $S_{c l}^{m}(E)$ the space of classical symbols of order $m$ on $E[7,29,30$. These spaces are reviewed in [2] in our framework.

Let $\chi \in \mathcal{C}^{\infty}\left(A^{*}\right)$ be a smooth function that is equal to 1 on $\left(A^{*}\right)_{r}$ and is equal to 0 outside $\left(A^{*}\right)_{2 r}$, for some $r<r_{0} / 3$. Then, following [2], we define

$$
a_{\chi}(D) u(x)=(2 \pi)^{-n} \int_{T^{*} M_{0}} e^{i \tau(x, y) \cdot \eta} \chi(x, \tau(x, y)) a(x, \eta) u(y) d \eta d y .
$$

This integral is an oscillatory integral with respect to the symplectic measure on $T^{*} M_{0}$ [7]. Alternatively, we can consider the measures on $M_{0}$ and on $T_{x}^{*} M_{0}$ defined by some choice of a metric on $A$ and then we integrate first along the fibers $T_{x}^{*} M_{0}$ and then along $M_{0}$.

Proposition 2.1. The map $\sigma_{t o t}: S_{1,0}^{m}\left(A^{*}\right) \rightarrow \Psi^{m}\left(M_{0}\right) / \Psi^{-\infty}\left(M_{0}\right)$,

$$
\sigma_{t o t}(a):=a_{\chi}(D)+\Psi^{-\infty}\left(M_{0}\right)
$$

is independent of the choice of the function $\chi \in \mathcal{C}_{c}^{\infty}\left((A)_{r}\right)$ used to define $a_{\chi}(D)$.

The space of all operators of the form $a_{\chi}(D)$, with $a \in S_{1,0}^{*}\left(A^{*}\right)$, is not closed under composition. In order to make it closed under composition, we are going to include more operators of order $-\infty$ in our calculus.

Any vector field $X \in \Gamma(A)$ generates a global flow $\Psi_{X}: \mathbb{R} \times M \rightarrow M$ because $X$ is tangent to all boundary faces of $M$ and $M$ is compact. Evaluating at $t=1$ yields a diffeomorphism

$$
\psi_{X}:=\Psi_{X}(1, \cdot): M \rightarrow M .
$$

We continue to assume that the injectivity radius $r_{0}$ of our fixed manifold with a Lie structure at infinity $\left(M, M_{0}, A\right)$ is strictly positive.

Definition 2.2. Fix $0<r<r_{0}$ and $\chi \in \mathcal{C}_{\mathrm{c}}^{\infty}\left((A)_{r}\right)$ such that $\chi=1$ in a neighborhood of $M$ in $A$. For $m \in \mathbb{R}$, the space $\Psi_{1,0, \mathcal{V}}^{m}\left(M_{0}\right)$ of pseudodifferential operators generated by the Lie structure at infinity $(M, A)$ is the linear space of operators $\mathcal{C}_{\mathrm{c}}^{\infty}\left(M_{0}\right) \rightarrow \mathcal{C}_{\mathrm{c}}^{\infty}\left(M_{0}\right)$ generated by $a_{\chi}(D), a \in S_{1,0}^{m}\left(A^{*}\right)$, and $b_{\chi}(D) \psi_{X_{1}} \ldots \psi_{X_{k}}$, $b \in S^{-\infty}\left(A^{*}\right)$ and $X_{j} \in \Gamma(A)$, for all $j$.

Similarly, the space $\Psi_{c l, \mathcal{V}}^{m}\left(M_{0}\right)$ of classical pseudodifferential operators generated by the Lie structure at infinity $(M, A)$ is obtained by using classical symbols $a$ in the construction above.

We also obtain that $\Psi_{1,0, \mathcal{V}}^{m}\left(M_{0}\right)$ and $\Psi_{c l, \mathcal{V}}^{m}\left(M_{0}\right)$ are algebras independent of the choices made in their definition. 
Theorem 2.3. The spaces $\Psi_{1,0, \mathcal{V}}^{m}\left(M_{0}\right)$ and $\Psi_{c l, \mathcal{V}}^{m}\left(M_{0}\right)$ are filtered algebras. They do not depend on the choice of the metric on $A$ and the function $\chi$ used to define $i t$, but depend, in general, on the Lie structure at infinity $(M, A)$ on $M_{0}$.

The fact that $\Psi_{1,0, \mathcal{V}}^{m}\left(M_{0}\right)$ and $\Psi_{c l, \mathcal{V}}^{m}\left(M_{0}\right)$ are filtered algebras means that

$$
\Psi_{1,0, \mathcal{V}}^{m}\left(M_{0}\right) \Psi_{1,0, \mathcal{V}}^{m^{\prime}}\left(M_{0}\right) \subseteq \Psi_{1,0, \mathcal{V}}^{m+m^{\prime}}\left(M_{0}\right), \quad \Psi_{c l, \mathcal{V}}^{m}\left(M_{0}\right) \Psi_{c l, \mathcal{V}}^{m^{\prime}}\left(M_{0}\right) \subseteq \Psi_{c l, \mathcal{V}}^{m+m^{\prime}}\left(M_{0}\right),
$$

for all $m, m^{\prime} \in \mathbb{C} \cup\{-\infty\}$.

The proof of Theorem 2.3 is obtained by realizing $\Psi_{1,0, \mathcal{V}}^{\infty}\left(M_{0}\right)$ as the homomorphic image of the algebra $\Psi_{1,0}^{\infty}(\mathcal{G})$ of pseudodifferential operators on a groupoid $\mathcal{G}$ integrating the Lie algebroid $A$ (that is, with $\Gamma(A)=\mathcal{V}$ ). This is possible due to the results of $[4,23,24]$. Note that this proof provides also an alternative definition of the algebras $\Psi_{1,0, \mathcal{V}}^{\infty}\left(M_{0}\right)$ and $\Psi_{c l, \mathcal{V}}^{\infty}\left(M_{0}\right)$. The advantage of our original definition, however, is that it is intrinsically formulated in terms of the geometry of $M$.

As for the usual algebras of pseudodifferential operators, we have the following basic property of the principal symbol.

Proposition 2.4. The principal symbol establishes isomorphisms

$$
\sigma^{(m)}: \Psi_{1,0, \mathcal{V}}^{m}\left(M_{0}\right) / \Psi_{1,0, \mathcal{V}}^{m-1}\left(M_{0}\right) \rightarrow S_{1,0}^{m}\left(A^{*}\right) / S_{1,0}^{m-1}\left(A^{*}\right)
$$

and

$$
\sigma^{(m)}: \Psi_{c l, \mathcal{V}}^{m}\left(M_{0}\right) / \Psi_{c l, \mathcal{V}}^{m-1}\left(M_{0}\right) \rightarrow S_{c l}^{m}\left(A^{*}\right) / S_{c l}^{m-1}\left(A^{*}\right) \simeq \mathcal{C}^{\infty}\left(S^{*} A\right),
$$

where $S^{*} A$ is the set of unit vectors in $A^{*}$.

We have the following boundedness result.

Proposition 2.5. Any operator $P \in \Psi_{1,0, \mathcal{V}}^{m}\left(M_{0}\right)$ defines a continuous linear operator on $\mathcal{C}_{c}^{\infty}\left(M_{0}\right)$ and $\mathcal{C}^{\infty}(M)$. If $m=0$, it also defines a bounded operator on $L^{2}\left(M_{0}\right)$.

Part (i) of the following result is an analog of a standard result about the $b$ calculus [18, whereas the second formula is the independence of diffeomorphisms of the algebras $\Psi_{c l, \mathcal{V}}^{\infty}\left(M_{0}\right)$, in the framework of manifolds with a Lie structure at infinity. Recall that if $X \in \Gamma(A)$, we have denoted by $\psi_{X}:=\Psi_{X}(1, \cdot): M \rightarrow M$ the diffeomorphism defined by integrating $X$ (and specializing at $t=1$ ).

Proposition 2.6. (i) Let $x$ be a defining function of some hyperface of $M$. Then

$$
x^{s} \Psi_{1,0, \mathcal{V}}^{m}\left(M_{0}\right) x^{-s}=\Psi_{1,0, \mathcal{V}}^{m}\left(M_{0}\right) \quad \text { and } \quad x^{s} \Psi_{c l, \mathcal{V}}^{m}\left(M_{0}\right) x^{-s}=\Psi_{c l, \mathcal{V}}^{m}\left(M_{0}\right)
$$

for any $s \in \mathbb{C}$.

(ii) Similarly,

$$
\psi_{X} \Psi_{1,0, \mathcal{V}}^{m}\left(M_{0}\right) \psi_{X}^{-1}=\Psi_{1,0, \mathcal{V}}^{m}\left(M_{0}\right) \quad \text { and } \quad \psi_{X} \Psi_{c l, \mathcal{V}}^{m}\left(M_{0}\right) \psi_{X}^{-1}=\Psi_{c l, \mathcal{V}}^{m}\left(M_{0}\right),
$$

for any $X \in \Gamma(A)$.

Let us notice that (ii) remains true for any diffeomorphism of $M_{0}$ that extends to an automorphism of $(M, A)$. Recall that an autormorphism of the Lie algebroid $(M, A)$ is a morphism of vector bundles $(\phi, \psi), \phi: M \rightarrow M, \psi: A \rightarrow A$, such that $\phi$ and $\psi$ are diffeomorphisms and

$$
\varrho_{\Gamma} \circ \psi_{\Gamma}=\phi_{*} \circ \varrho_{\Gamma}
$$


where $\varrho_{\Gamma}: \Gamma(A) \rightarrow \Gamma(T M)$ and $\psi_{\Gamma}: \Gamma(A) \rightarrow \Gamma(A)$ are the maps defined by the anchor map $\varrho$ and $\psi$, respectively, and $\phi_{*}: \Gamma(T M) \rightarrow \Gamma(T M)$ is given by the differential of $\phi$.

A proof of the above proposition can be obtained using the corresponding results for the algebras of pseudodifferential operators on $\mathcal{G}$.

The proof of the following proposition relies on the Campbell-Hausdorff formula.

Proposition 2.7. Let $X \in \Gamma(A)$ and denote by $a_{X}(\xi)=\xi(X)$ the associated linear function on $A^{*}$. Then $a_{X} \in S^{1}\left(A^{*}\right)$ and $a_{X}(D)=-\imath X$. Moreover,

$$
\left\{a_{\chi}(D), a=\text { polynomial in each fiber }\right\}=\operatorname{Diff}_{\mathcal{V}}^{*}\left(M_{0}\right) .
$$

From this we obtain the following result.

Theorem 2.8. Let Diff $\left(M_{0}\right)$ be the algebra of all differential operators on $M_{0}$. Then

$$
\Psi_{1,0, \mathcal{V}}^{\infty}\left(M_{0}\right) \cap \operatorname{Diff}\left(M_{0}\right)=\operatorname{Diff}_{\mathcal{V}}^{*}\left(M_{0}\right) .
$$

Since $\operatorname{Diff}_{\mathcal{V}}^{*}\left(M_{0}\right) \subset \Psi_{c l, \mathcal{V}}^{\infty}\left(M_{0}\right)$, it also follows that

$$
\Psi_{c l, \mathcal{V}}^{\infty}\left(M_{0}\right) \cap \operatorname{Diff}\left(M_{0}\right)=\operatorname{Diff}_{\mathcal{V}}^{*}\left(M_{0}\right) .
$$

Thus the algebras $\Psi_{1,0, \mathcal{V}}^{\infty}\left(M_{0}\right)$ and $\Psi_{1,0, \mathcal{V}}^{\infty}\left(M_{0}\right)$ are microlocalizations of Diff* ${ }_{\mathcal{V}}^{*}\left(M_{0}\right)$, which, together with the other properties of these algebras stated above, shows that our constructions solve a conjecture from [17].

\section{REFERENCES}

[1] B. Ammann, R. Lauter, and V. Nistor. On the Riemannian geometry of manifolds with a Lie structure at infinity. To appear in Int. J. Math. and Math. Sci.

[2] B. Ammann, R. Lauter, and V. Nistor. Pseudodifferential operators on manifolds with a Lie structure at infinity. Preprint, December 2002.

[3] B. Ammann, R. Lauter, V. Nistor, and A. Vasy. Complex powers and non-compact manifolds. To appear in Commun. Partial Differential Equations.

[4] M. Crainic and R. L. Fernandes. Integrability of Lie brackets. Ann. of Math. 157 (2003), $575-620$.

[5] C. Epstein, R. B. Melrose, and G. Mendoza. The Heisenberg algebra, index theory and homology. In preparation.

[6] C. Epstein, R. B. Melrose, and G. Mendoza. Resolvent of the Laplacian on strictly pseudoconvex domains. Acta Math. 167 (1991), 1-106. MR 92i:32016

[7] L. Hörmander. The analysis of linear partial differential operators, vol. 3. Pseudo-differential operators, volume 274 of Grundlehren der Mathematischen Wissenschaften. Springer-Verlag, Berlin-Heidelberg-New York, 1985. MR 87d:35002a

[8] M. Karoubi. Homologie cyclique et K-theorie. Astérisque 149 (1987), 1-147. MR 89c:18019

[9] R. Lauter. Pseudodifferential analysis on conformally compact spaces. Mem. Amer. Math. Soc., 163, 2003.

[10] R. Lauter and S. Moroianu. Fredholm theory for degenerate pseudodifferential operators on manifolds with fibered boundaries. Commun. Partial Differential Equations 26 (2001), 233283. MR 2002e:58052

[11] R. Lauter and V. Nistor. Analysis of geometric operators on open manifolds: a groupoid approach. In N. P. Landsman, M. Pflaum, and M. Schlichenmaier, editors, Quantization of Singular Symplectic Quotients, volume 198 of Progress in Mathematics, pages 181-229. Birkhäuser, Basel-Boston-Berlin, 2001. MR 2003e:53003

[12] J. N. Mather. Stratifications and mappings. In Dynamical systems (Proc. Sympos., Univ. Bahia, Salvador, 1971), pages 195-232. Academic Press, New York, 1973. MR 51:4306

[13] R. Mazzeo. Elliptic theory of differential edge operators. I. Commun. Partial Differ. Equations 16 (1991), 1615-1664. MR 93d:58152 
[14] R. Mazzeo and R. B. Melrose. Meromorphic extension of the resolvent on complete spaces with asymptotically constant negative curvature. J. Funct. Anal. 75 (1987), 260-310. MR 89c:58133

[15] R. Mazzeo and R. B. Melrose. Pseudodifferential operators on manifolds with fibred boundaries. Asian J. Math. 2 (1998), 833-866. MR 2000m:58046

[16] R. B. Melrose. Transformation of boundary value problems. Acta Math. 147 (1981), 149-236. MR 83f: 58073

[17] R. B. Melrose. Pseudodifferential operators, corners and singular limits. In Proceeding of the International Congress of Mathematicians, Kyoto, pages 217-234, Berlin-Heidelberg-New York, 1990. Springer-Verlag. MR 93j:58131

[18] R. B. Melrose. The Atiyah-Patodi-Singer index theorem. Research Notes in Mathematics (Boston, Mass.). 4. Wellesley, MA: A. K. Peters, Ltd., 1993. MR 96g:58180

[19] R. B. Melrose. Geometric scattering theory. Stanford Lectures. Cambridge University Press, Cambridge, 1995. MR 96k:35129

[20] R. B. Melrose. Fibrations, compactifications and algebras of pseudodifferential operators. In L. Hörmander and A. Mellin, editors, Partial Differential Equations and Mathematical Physics, pages 246-261, 1996. MR 98j:58117

[21] R. B. Melrose. Geometric optics and the bottom of the spectrum. In F. Colombini and N. Lerner, editors, Geometrical optics and related topics, volume 32 of Progress in nonlinear differential equations and their applications. Birkhäuser, Basel-Boston-Berlin, 1997.

[22] R. B. Melrose and G. Mendoza. Elliptic operators of totally characteristic type. MSRI Preprint 1983.

[23] V. Nistor. Groupoids and the integration of Lie algebroids. J. Math. Soc. Japan 52 (2000), 847-868. MR 2002e:58035

[24] V. Nistor, A. Weinstein, and P. Xu. Pseudodifferential operators on groupoids. Pacific J. Math. 189 (1999), 117-152. MR 2000c:58036

[25] C. Parenti. Operatori pseudodifferentiali in $\mathbb{R}^{n}$ e applicazioni. Ann. Mat. Pura Appl. 93 (1972), 359-389. MR 55:10838

[26] E. Schrohe and B.-W. Schulze. Boundary value problems in Boutet de Monvel's algebra for manifolds with conical singularities. II. In Boundary value problems, Schrödinger operators, deformation quantization, volume 8 of Math. Top., pages 70-205, Akademie Verlag, Berlin, 1995. MR 96k:58001

[27] B.-W. Schulze. Boundary value problems and singular pseudo-differential operators. WileyInterscience Series in Pure and Applied Mathematics. Chichester: John Wiley \& Sons., 1998. MR 99m:35281

[28] M. A. Shubin. Spectral theory of elliptic operators on noncompact manifolds. Astérisque, 207:5, 35-108, 1992. Méthodes semi-classiques, Vol. 1 (Nantes, 1991). MR 94h:58175

[29] M. Taylor. Pseudodifferential operators, volume 34 of Princeton Mathematical Series. Princeton University Press, Princeton, NJ, 1981. MR 82i:35172

[30] M. Taylor. Partial differential equations, volumes I-III of Applied Mathematical Sciences. Springer-Verlag, New York, 1995-1997. MR 98b:35002a: MR 98b:35002b: MR 98b:35003 MR 98b:35001

[31] A. Vasy. Propagation of singularities in many-body scattering. Ann. Sci. École Norm. Sup. (4) 34 (2001), 313-402. MR 2002e:35255

[32] J. Wunsch. Propagation of singularities and growth for Schrödinger operators. Duke Math. J. 98 (1999), 137-186. MR 2000h:58054

Universität Hamburg, Fachbereich 11-Mathematik, Bundesstrasse 55, D-20146 HamBURG, GERMANY

E-mail address: ammann@berndammann.de

Universität Mainz, Fachbereich 17-Mathematik, D-55099 Mainz, Germany

E-mail address: lauter@mathematik.uni-mainz.de, lauterr@web.de

Mathematics Department, Pennsylvania State University, University Park, PA 16802

E-mail address: nistor@math.psu.edu 